\title{
Studying the dynamic parameters of the plasma pellet produced by helium jet in the presence of different ambient gases
}

\author{
S Serajuddin Razivizadeh and H R Qomi Marzdashti
}

Laser and Plasma Research Institute, Shahid Beheshti University, Tehran, Iran

E-mail: h-gmdashty@sbu.ac.ir

(Received 20 November 2018 ; in final form 16 August 2019)

\begin{abstract}
In this paper, the dynamic parameters of a plasma pellet (guided ionization wave) in the presence of oxygen, nitrogen and dry air gases are investigated. The plasma pellet dynamics produced by a helium plasma jet at atmospheric pressure with the frequency of $30 \mathrm{kHz}$ from the moment of departure of the jet tube to the moment of complete destruction in the surrounding ambient environment is recorded and measured by an ultra-fast camera (ICCD). Influenced by ambient gases, plasma pellets have different velocity, size, and length of diffusion in each of the ambient gases. Plasma pellet velocity in oxygen and dry air is higher than that in nitrogen, reaching up to $18 \mathrm{~km} / \mathrm{second}$. The maximum length of plasma pellet emission in nitrogen and dry air is about $12 \mathrm{~mm}$. Helium plasma jet spectrum has also been measured to investigate the chemical species produced in different ambient gases. The results of these experiments showed the effect of the ambient gases, especially the role of oxygen molecules on plasma pellet emission.
\end{abstract}

Keywords: plasma pellet, plasma Jet, guided ionizing wave, ultrafast camera, ambient gas, emission length

For full article, refer to the Persian section 